\title{
Initiation of turbulence and chaos in non-equilibrium inhomogeneous media: Wave beams
}

\author{
P.S. Landa \\ Physics Department, Lomonosov Moscow State University, Moscow 119899, Russia
}

\section{P.V.E. McClintock}

Department of Physics, Lancaster University, Lancaster, LA1 4YB, United Kingdom

\begin{abstract}
We show that wave excitation and propagation in an inhomogeneous medium leads to the simultaneous appearance of a huge number of waves with different phase velocities. This phenomenon arises in any medium with inhomogeneous parameters, e.g. in fluid jets where the inhomogeneity appears as a result of the boundary layer. Because of fluctuations (however small) the waves become randomised, i.e. turbulence develops. We demonstrate that the eigenvalues depend essentially on the frequency of the perturbation and on the distance from the initial section of a jet or wave beam. We show how to find the continuous set of eigenvalues - complex wave numbers - and corresponding eigenfunctions for any given frequency. The implication of these results is that the transition to turbulence occurs, not through the excitation of a gradually increasing number of waves, as commonly supposed, but by the simultaneous excitation of a continuous wave spectrum.
\end{abstract}

PACS numbers: $47.27 . \mathrm{wg}, 47.27 . \mathrm{Cn}, 47.20 . \mathrm{Ft}, 47.27 . \mathrm{ed}$

\section{Introduction}

Turbulence is a well-known phenomenon that arises in continuous media $[1,2,3,4]$ under conditions such that they become unstable. The best-known example is of course fluid flow with a large enough Reynolds number $[5,6,7,8,9,10]$. Other instances include kink waves [11], waves of burning [12, 13, 14, 15, 16, 17, 18], and waves in excitable media $[19,20,21,22,23,24,25]$.

Most of these works consider the propagation of plane waves in homogeneous media, an idealization that yields the simplest equations. In reality, however, plane waves often fail to provide a realistic description. Furthermore, the propagation medium may not be homogeneous and the variables describing wave processes are always bounded in space. Where the wave front has a narrow characteristic of directionality [26] propagation occurs as a wave beam $[27,28,26]$; a submerged jet [9] can be considered as a particular case of a wave beam. 
The excitation and propagation of waves is usually considered only for homogeneous media. The best known examples arise in the derivation of the Korteweg-de Vries and Ginsburg-Landau equations (see, for example, [29]). In our earlier consideration of the transition to turbulence in slowly-diverging subsonic submerged jets [30] we considered a medium where an inhomogeneity appears because of the dependence of the flow velocity on coordinates. We did not appreciate, however, that such an inhomogeneity may lead to entirely new effects. What we found was that, for large Reynolds numbers, ordinary methods of solving even the linearized Navier-Stokes equations yield random results on account of instability. We therefore used a WKB-like asymptotic expansion method for approximate solution of the complex, linear, fourth-order, differential equation that describes small deviations from the steady-state stream function. The equation contains a large parameter proportional to the root-mean-square of the Reynolds number. This method allowed us to find the complex eigenvalues and eigenfunctions but, in doing so, we found that the eigenvalues depend on the point where the condition for the vanishing solution is applied. By moving this point we obtained a continuous series of eigenvalues.

We therefore decided to establish whether the same phenomenon also arises in other inhomogeneous wave systems. As an example, we have chosen to consider a modified form of the Klein-Gordon equation (KGE) - an equation that is already well-known in mathematical physics. It is very close to the standard wave equation, and differs from it only in that it takes account of dispersion. It goes without saying that we could in principle have considered any equation describing wave propagation but, based on the universality of the theory of oscillations and waves [31, 32], we are confident that the results would be essentially the same as those that we discuss below.

In what follows we investigate in detail the behavior of the wave beams generated by this equation near the threshold of turbulence, where we can use a linear approximation. Section 2 contains the meat of the paper. In section 2(a) we introduce the KGE in its generalized form and discuss its properties, and in section 2(b) we seek generative solutions of the KGE and consider the implications of the results obtained. In section 3 we summarise our findings and draw conclusions.

\section{Propagation of a wave beam in a two-dimensional inhomogeneous medium described by a generalized Klein-Gordon equation}

\subsection{Generalized Klein-Gordon equation}

We consider a wave beam propagating in the $x$ direction, described by

$$
\begin{aligned}
& \frac{\partial^{2} w}{\partial t^{2}}-U^{2}(x, y) \frac{\partial^{2} w}{\partial x^{2}}-V^{2}(x) \frac{\partial^{2} w}{\partial y^{2}}+\alpha_{x} \frac{\partial w}{\partial x}-\alpha_{y} \frac{\partial w}{\partial y}+\beta w \\
& =\epsilon(f(w, \partial w / \partial x))
\end{aligned}
$$

which we will call the generalized Klein-Gordon equation. Here $U(x, y)$ is the wave velocity in the $x$ direction and $V(x)$ is the wave velocity in the $y$ direction, $\alpha_{x}$ and 
$\alpha_{y}$ are are coefficients describing the stability of the medium in the $x$ and $y$ directions respectively, $\beta$ is the dispersion coefficient for the medium, $x$ is the distance from the beam initial section, $y$ is the distance from the beam axes, $f(w, \partial w / \partial x)$ is a nonlinear function, and $\epsilon$ is a conditional small parameter describing the degree of nonlinearity. Eq. 1 describes processes in an inhomogeneous medium whose parameters depend on coordinates. In the particular case when $\alpha_{x}=\alpha_{y}=0$, it describes the oscillations of an inhomogeneous membrane lying on an elastic base.

We note that the presence of inhomogeneities neither facilitates nor impedes the transition to turbulent behavior, but it changes the way in which the turbulence develops. Quite generally, the onset of turbulence near the threshold [33] occurs through the excitation of a gradually increasing number of modes to three, corresponding to a three-dimensional torus in phase space. Further this torus either collapses or a chaotic attractor is born on the torus [34], and turbulence subsequently develops via different routes in different systems [33].

For definiteness we set

$$
U(x, y)=\frac{1-U_{0}(x)}{2}\left[1-\tanh \left(q \frac{|y|-1}{\delta_{0}(x)}\right)\right]+U_{0}(x),
$$

where $U_{0}(x)$ is the value of $U(x, y)$ for $|y| \rightarrow \infty, \delta_{0}(x)$ is the boundary layer thickness that is expected to increase with increasing $x$. We set

$$
U_{0}(x)=\frac{\delta_{00}}{1+\mu x}, \quad \delta_{0}(x)=\delta_{00}(1+x)
$$

where $\delta_{00}=0.01, \mu$ is another small parameter characterizing the rate of change of $U(x, y)$ as $x$ varies. It leads to the beam width increasing as the beam propagates. Examples of the dependence of $U$ on $y$ for particular values of $x$ are shown in Fig. 1. The dependence of the wave velocity $U(x, y)$ on coordinate corresponds to a type of inhomogeneity that often arises in reality - namely, where the medium under consideration consists of two different parts separated by an intermediate layer.

Provided that the wave dispersion in the medium is large enough, we can use a quasi-linear approximation for derivation of an equation describing the behavior of a bounded beam and apply a well-known perturbation technique: the van der Pol, asymptotic, average [35, 36, 29, 37]. In what follows we also apply the asymptotic Krylov-Bogolyubov method for spatially extended systems [29]. We therefore seek a solution of Eq. (1) in the form of a series in $\epsilon$ :

$$
w(x, y, t)=w_{0}(x, y, t)+\epsilon w_{1}(x, y, t)+\epsilon^{2} w_{2}(x, y, t)+\ldots,
$$

where $w_{0}(x, y, t)$ is a generative solution of Eq. (1), and $w_{1}(x, y, t), w_{2}(x, y, t), \ldots$ are unknown functions.

\subsection{Generative solution}

The generative solution of Eq. (1) is described by the linear equation

$$
\frac{\partial^{2} w_{0}}{\partial t^{2}}-U^{2}(x, y) \frac{\partial^{2} w_{0}}{\partial x^{2}}-V^{2}(x) \frac{\partial^{2} w_{0}}{\partial y^{2}}+\alpha_{x} \frac{\partial w_{0}}{\partial x}-\alpha_{y} \frac{\partial w_{0}}{\partial y}+\beta w_{0}=0
$$




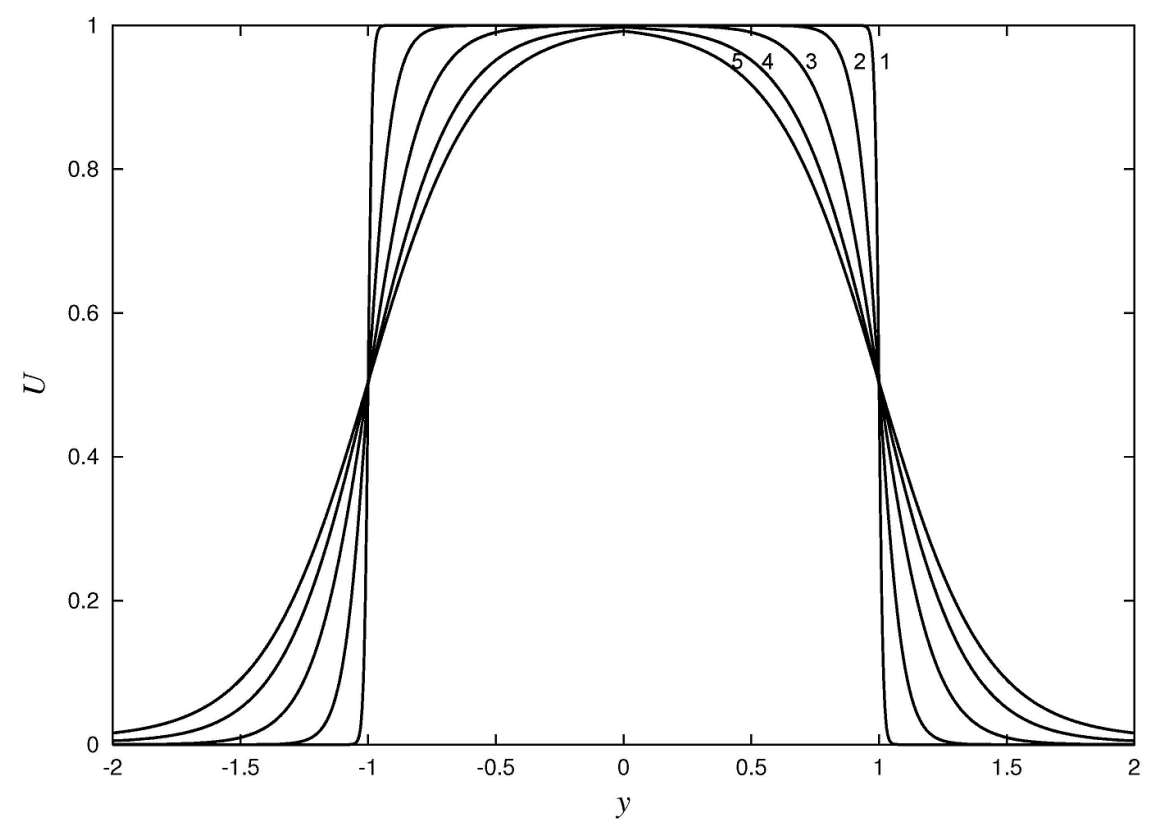

Figure 1. Examples of the dependences $U$ on $y$ for $q=2.5, U_{0}(x)=\delta_{00} /(1+0.01 x)$, $\delta_{0}(x)=\delta_{00}(1+x), \delta_{00}=0.01, x=0(1), x=2(2), x=5(3), x=8(4)$, and $x=10$ $(5)$

As in [30] we seek a partial solution of Eq. (5) in the form of a sum of wavesł of frequency $\omega$, amplitude $A(\omega, y)$ and complex wave number $Q(\omega, x)=K(\omega, x)+i \Gamma(\omega, x)$, where $K(\omega, x)=\omega / v_{\mathrm{ph}}(\omega, x)$ is the real wave number and $v_{\mathrm{ph}}(\omega, x)$ is the wave phase velocity. So,

$$
w_{0}(t, x, y)=\frac{1}{2 \pi} \int_{-\infty}^{\infty} A(\omega, y) \exp \left[i\left(\omega t-\int_{0}^{x} Q(\omega, x) d x\right)\right] d \omega .
$$

We rewrite (6) as

$$
w_{0}(t, x, y)=\frac{1}{2 \pi} \int_{-\infty}^{\infty} A(\omega, x, y) e^{i \omega t} d \omega
$$

where

$$
A(\omega, x, y)=A(\omega, y) \exp \left(-i \int_{0}^{x} Q(\omega, x) d x\right) .
$$

It follows from (7) that $A(\omega, x, y)$ is the Fourier transform of function $w_{0}(t, x, y)$.

Taking account of (7) and (8) and imposing the condition of the wave damping in the direction of positive $y$, Eq. (5) for $A(\omega, x, y)$ becomes

$$
V^{2}(x) \frac{\partial^{2} A(\omega, x, y)}{\partial y^{2}}+\alpha_{y} \operatorname{sign} y \frac{\partial A(\omega, x, y)}{\partial y}+\left(\omega^{2}+i \alpha_{x} Q(\omega, x)\right.
$$

$\ddagger$ Note that in [30] the frequency was expressed in terms of the Strouhal number, as is customary in aerodynamics. 


$$
\left.-U^{2}(x, y) Q^{2}(\omega, x)-\beta-i U^{2}(x, y) \frac{\partial Q(\omega, x)}{\partial x}\right) A(\omega, x, y)=0 .
$$

As can be seen from Fig. 1, the velocity $U(x, y)$ changes slowly as $x$ increases, so that we can set

$$
\frac{\partial Q(\omega, x)}{\partial x} \sim \mu
$$

Hence we can represent $A(\omega, x, y)$ and $Q(\omega, x)$ as series:

$$
\begin{aligned}
& A(\omega, x, y)=A_{0}(\omega, x, y)+\mu A_{1}(\omega, x, y)+\ldots \\
& Q(\omega, x)=Q_{0}(\omega, x)+\mu Q_{1}(\omega, x)+\ldots
\end{aligned}
$$

Substituting (11) into Eq. (9) and equating the coefficients of the same power of $\mu$, we obtain for $A_{0}(\omega, x, y), A_{1}(\omega, x, y), \ldots$ the following equations:

$$
\begin{aligned}
L_{0} A_{0}(\omega, x, y)= & 0, \\
L_{0} A_{1}(\omega, x, y)= & {\left[i U^{2}(x, y) \frac{\partial Q_{0}(\omega, x)}{\partial x}-\left(i \alpha_{x}+2 Q_{0}(\omega, x) U^{2}(x, y)\right) Q_{1}(\omega, x)\right] } \\
& \times A_{0}(\omega, x, y), \\
& \ldots \ldots \ldots,
\end{aligned}
$$

where

$$
L_{0}=V^{2}(x) \frac{\partial^{2}}{\partial y^{2}}+\alpha_{y} \operatorname{sign} y \frac{\partial}{\partial y}+\omega^{2}-U^{2}(x, y) Q_{0}^{2}(\omega, x)+i \alpha_{x} Q_{0}(\omega, x)-\beta(
$$

is a differential operator.

In view of (14), and the boundary conditions for the function $A_{0}(\omega, x, y)$,

$$
A_{0}(\omega, x, \pm \infty)=0,\left.\quad \frac{\partial A_{0}(\omega, x, y)}{\partial y}\right|_{y= \pm \infty}=0 .
$$

Eq. (12) describes a not-self-adjoint boundary-value problem, whose solution allows us to find the complex eigenvalues of $Q_{0}(\omega, x)$ together with the corresponding eigenfunctions. Similar boundary-value problems were solved analytically in [38] and numerically in [30].

In the derivation of the Ginsburg-Landau equation we noted [29] that a plane wave may be taken as a generative solution, but it is valid only while the widening of wave front remains insignificant. In contrast, because we set the boundary conditions for $A_{0}(\omega, x, y)$ to vanish at large values of $y$, our present approach is valid even for wave beams that are significantly diverging.

We will solve our boundary-value problem by one of the same ways as in [30]. Given (14), any solution of Eq. (12) can be represented as a linear combination of even and odd solutions. We restrict ourselves to odd solutions.

It follows from (2), and in view of (14), that for small and large $y$ the differential equation (12) becomes an equation with coefficients independent of $y$ :

$$
\begin{array}{ll}
V^{2}(x) \frac{\partial^{2} A_{0}}{\partial y^{2}}+\alpha_{y} \frac{\partial A_{0}}{\partial y} \operatorname{sign} y-k_{0}^{2}(\omega, x) A_{0}=0, & \text { for }|y| \ll 1, \\
V^{2}(x) \frac{\partial^{2} A_{0}}{\partial y^{2}}+\alpha_{y} \frac{\partial A_{0}}{\partial y} \operatorname{sign} y-k_{1}^{2}(\omega, x) A_{0}=0 & \text { for }|y| \gg 1,
\end{array}
$$


where

$$
\begin{aligned}
& k_{0}(\omega, x)=\sqrt{Q_{0}^{2}(\omega, x)-\omega^{2}+\beta-i \alpha_{x} Q_{0}(\omega, x)}, \\
& k_{1}(\omega, x)=\sqrt{U_{0}^{2}(x) Q_{0}^{2}(\omega, x)-\omega^{2}+\beta-i \alpha_{x} Q_{0}(\omega, x)} .
\end{aligned}
$$

Odd partial solutions of Eqs. (16) satisfying the boundary conditions (15) are

$$
A_{0}(\omega, x, y)= \begin{cases}C_{1} \exp \left(-\frac{\alpha_{y}|y|}{2 V^{2}(x)}\right) \sinh \left(\sqrt{\left.\frac{\alpha_{y}^{2}}{4 V^{4}(x)}+\frac{k_{0}^{2}(\omega, x)}{V^{2}(x)} y\right)}\right. & \text { for }|y| \ll y_{0}, \\ C_{2} \exp \left[-\left(\frac{\alpha_{y}}{2 V^{2}(x)}+\sqrt{\left.\frac{\alpha_{y}^{2}}{4 V^{4}(x)}+\frac{k_{1}^{2}(\omega, x)}{V^{2}(x)}\right)}|y|\right]\right. & \text { for }|y| \gg y_{0} .\end{cases}
$$

In view of (14), it then follows that an odd solution of Eq. (12), for $y \leq y_{0}$ may be found numerically for the initial conditions

$$
A_{0}(\omega, x, 0)=0,\left.\quad \frac{\partial A_{0}(\omega, x, y)}{\partial y}\right|_{y=0}=\sqrt{\frac{\alpha_{y}^{2}}{4 V^{4}(x)}+\frac{k_{0}^{2}(\omega, x)}{V^{2}(x)}} .
$$

Since $\partial A_{0}(\omega, x, y) /\left.\partial y\right|_{y=0}$ is positive, this solution, which we denote as $A_{01}(\omega, x, y)$, grows with increasing $y$, at least for small $y$.

To satisfy the boundary conditions (15), we must find a partial solution of Eq. (12) subject to (14) that decreases in absolute value as $y$ increases. We can find such a solution by solving Eq. (12) with boundary conditions

$$
A_{0}\left(\omega, x, y_{e}\right)=1,\left.\quad \frac{\partial A_{0}}{\partial y}\right|_{y=y_{e}}=-\left(\frac{\alpha_{y}}{2 V^{2}(x)}+\sqrt{\frac{\alpha_{y}^{2}}{4 V^{4}(x)}+\frac{k_{1}^{2}(\omega, x)}{V^{2}(x)}}\right)
$$

where $y_{e}$ is some sufficiently large value of $|y|$. We denote this solution $A_{02}(\omega, x, y)$.

So, the solutions of Eq. (12) satisfying the required boundary conditions are

$$
A_{0}(\omega, x, y)= \begin{cases}C_{1}(\omega, x) A_{01}(\omega, x, y) & \text { for } 0 \leq y \leq y_{0} \\ C_{2}(\omega, x) A_{02}(\omega, x, y) & \text { for } y \geq y_{0}\end{cases}
$$

where $C_{1}$ and $C_{2}$ are arbitrary functions, and $y_{0}$ is some point where we sew solutions $A_{01}(\omega, x, y)$ and $A_{02}(\omega, x, y)$. The sewing conditions are

$$
\begin{aligned}
& C_{1}(\omega, x) A_{01}\left(\omega, x, y_{0}\right)-C_{2}(\omega, x) A_{02}\left(\omega, x, y_{0}\right)=0 \\
& \left.C_{1}(\omega, x) \frac{\partial A_{01}}{\partial y}\right|_{y=y_{0}}-\left.C_{2}(\omega, x) \frac{\partial A_{02}}{\partial y}\right|_{y=y_{0}}=0 .
\end{aligned}
$$

The system (22) has a nontrivial solution if its determinant is equal to zero, i.e. if

$$
\begin{aligned}
D\left(Q_{0}(\omega, x), y_{0}\right)= & \left.A_{01}\left(\omega, x, y_{0}\right) \frac{\partial A_{02}(\omega, x, y)}{\partial y}\right|_{y=y_{0}} \\
& -\left.A_{02}\left(\omega, x, y_{0}\right) \frac{\partial A_{01}(\omega, x, y)}{\partial y}\right|_{y=y_{0}}=0 .
\end{aligned}
$$


The complex equation (23) allows us to calculate eigenvalues of the complex wave number $Q_{0}(\omega, x)$ for given $\omega, x$ and $y_{0}$.

Eqs. (22) enable us to find the relationship between $C_{1}(\omega, x)$ and $C_{2}(\omega, x)$ :

$$
\frac{C_{2}(\omega, x)}{C_{1}(\omega, x)}=\frac{A_{01}\left(\omega, x, y_{0}\right)}{A_{02}\left(\omega, x, y_{0}\right.} .
$$

It is known [39] that in many cases the numerical solution of $n$ equations

$$
F_{i}\left(z_{1}, z_{2}, z_{n}\right)=0, \quad i=1 \ldots n
$$

with $n$ unknowns $z_{1}, z_{2}, \ldots, z_{n}$ may be reduced to the problem of searching for a global minimum in the modulus of some functional $\Phi\left(F_{i}\right)$. For our problem $Q_{0}(\omega, x)=$ $K_{0}(\omega, x)+i \Gamma_{0}(\omega, x)$, where $K_{0}(\omega, x)$ and $\Gamma_{0}(\omega, x)$ are roots of two equations

$$
\operatorname{Re}\left(D\left(Q_{0}(\omega, x)\right), y_{0}\right)=0, \quad \operatorname{Im}\left(D\left(Q_{0}(\omega, x)\right), y_{0}\right)=0 .
$$

Different methods have been described for numerical solution of similar equations, for example in [40,39]. All of these methods are based on the supposition that the functional $\left|D\left(Q_{0}(\omega, x), y_{0}\right)\right|$ changes monotonically with changes in $\left|Q_{0}(\omega, x)\right|$. However, our calculations show that this is not in fact the case. We have therefore used a trialand-error method.

In the general case, an analytic solution of the equations

$$
\operatorname{Re}\left(D\left(Q_{0}(\omega, x)\right), y_{0}\right)=0, \quad \operatorname{Im}\left(D\left(Q_{0}(\omega, x)\right), y_{0}\right)=0
$$

is impossible. We therefore solve them numerically. Setting $\omega, x, v_{\text {pho }}(\omega, x)$ and the sewing point $y_{0}$ we calculate $\operatorname{Re}\left(D\left(Q_{0}(\omega, x)\right)\right)$ and $\operatorname{Im}\left(D\left(Q_{0}(\omega, x)\right)\right)$ as a functions of $\Gamma_{0}$ and find their point intersection $\Gamma_{0}^{*}$. Further for $\Gamma_{0}=\Gamma_{0}^{*}$ we vary $v_{\mathrm{ph} 0}(\omega, x)$ until $\left|D\left(Q_{0}(\omega, x)\right)\right|$ does not become minimal. After that we again find the point $\Gamma_{0}^{*}$, and so on. If the values $\Gamma_{0}(\omega, x)$ and $v_{\mathrm{ph} 0}(\omega, x)$ found by this manner exist, then they are the eigenvalues we seek. Our calculations show that they exist only above a certain value of $\omega$ which decreases as $x$ increases. We denote this value as $\omega_{\text {cr }}$. The dependence of $\omega_{\text {cr }}$ on $x$ is illustrated in Fig. 2: the waves under consideration are excited only for $\omega>\omega_{\text {cr }}(x)$.

We have repeated the same procedure for a wide range of $\omega, x$ and $y_{0}$ values. In doing so, we found that the values of $\Gamma_{0}(\omega, x)$ and $v_{\mathrm{ph} 0}(\omega, x)$ are practically independent of the sewing point. The dependences of the eigenvalues of the gain factor $\Gamma_{0}$, wave phase velocity $v_{\text {ph } 0}$, wave number $K_{0}$ and wave length $\lambda=2 \pi / K_{0}$ on the wave perturbation frequency $\omega$ for certain values of $x$ are shown in Fig. 3. We see that the gain factor, phase velocity and the wave length all decrease as the wave frequency increases. Note that the phase velocity depends strongly on frequency, thus implying that these waves are significantly dispersive. Thus, for any given position $x$ we have found a continuous set of eigenvalues for the frequency and phase velocity; $c f$. finite homogeneous media where only a discrete set of eigenvalues is possible.

To clarify how the wave changes with increasing distance from the initial section of the beam, we construct the dependences of the gain factor, wave phase velocity wave 


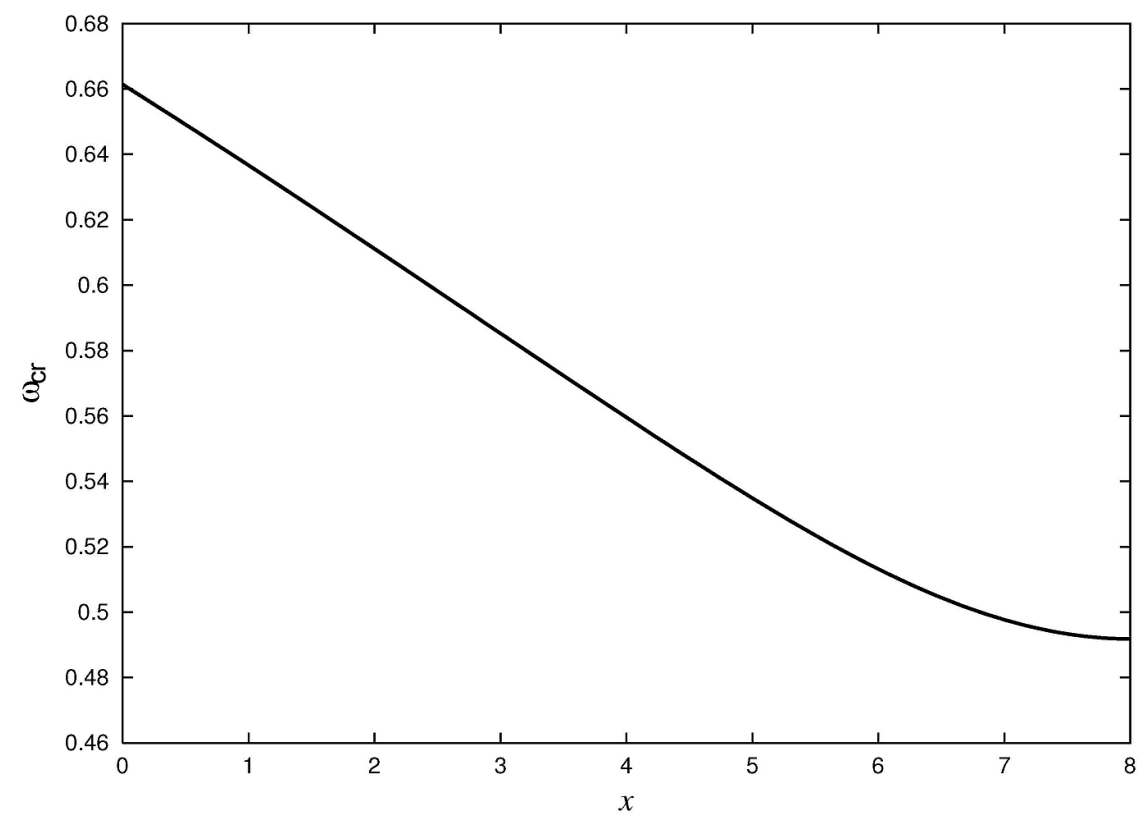

Figure 2. The dependence of $\omega_{\text {cr }}$ on the distance $x$

number and wave length on this distance, as shown in Fig. 4. It can be seen that, with increasing $x$, the gain factor decreases fast with increasing distance, whereas wave the phase velocity, wave number and wave length are characterised by weaker dependences.

It is of interest to note that the gain factor $\Gamma_{0}$ depends only weakly on the coefficient $\alpha_{x}$ responsible for the medium's stability in the $x$ direction. For example, at $x=4$ the value of $\Gamma_{0}$ changes only from 0.3875 to 0.3375 when $\alpha_{x}$ is halved (from 0.2 to 0.1 ).

To find the eigenfunctions corresponding to the eigenvalues $Q_{0}(\omega, x)$, we must calculate the functions $A_{01}(\omega, x, y), A_{02}(\omega, x, y)$ for even eigenvalues of $Q_{0}(\omega, x)$, sewing them at the point $y_{0}$. The functions

$$
A_{0}(\omega, x, y)=C(\omega, x) \begin{cases}A_{01}(\omega, x, y) & \text { for } 0 \leq y \leq y_{0}, \\ \frac{A_{01}\left(\omega, x, y_{0}\right)}{A_{02}\left(\omega, x, y_{0}\right)} A_{02}(\omega, x, y) & \text { for } y \geq y_{0}\end{cases}
$$

are the eigenfunctions sought.

Knowing $Q_{0}(\omega, x)$ and the expression $C$ we can calculate the evolution of the wave power spectrum in the linear approximation. To do so we must set the power spectra at the input section. If we suppose that the spectral density of the input disturbances are independent of frequency and equal $\kappa_{0}$, then the spectral density $\kappa(\omega, x, y)$ of the process $w_{0}(t, x, y)$, defined by $(7)$, is described by expression

$$
\kappa(\omega, x, y)=\mid A_{0}\left(\omega, x,\left.y\right|^{2},\right.
$$

where $A_{0}(\omega, x, y$ is defined by $(27)$.

We emphasize that the results are obtained from the linear theory without taking account of nonlinear phenomena such as the pairing of vortices. Processes of this kind, 

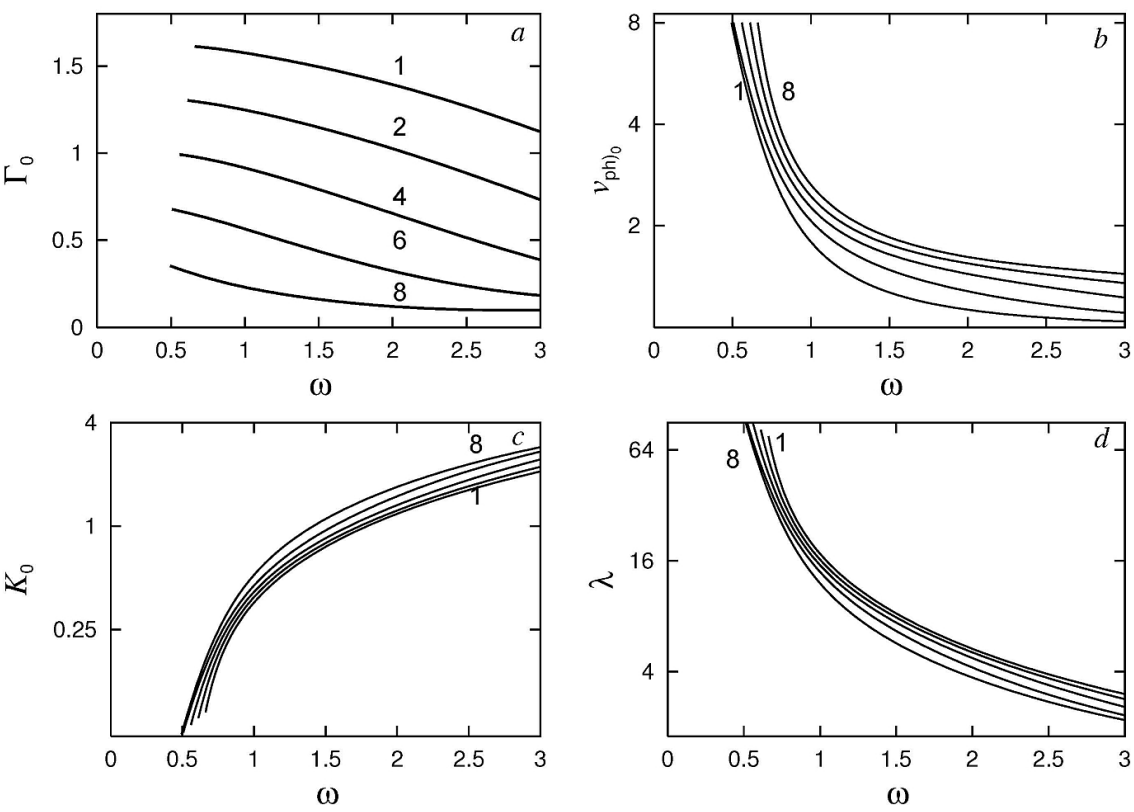

Figure 3. Dependences of the eigenvalues of the gain factor $\Gamma_{0}(a)$ on phase velocity $v_{\text {ph0 }}(b)$, wave number $K_{0}(c)$ and wave length $\lambda(d)$ on the wave perturbation frequency $\omega$ for $x=0(1), x=2(2), x=4(4), x=6(6), x=8$ (8)
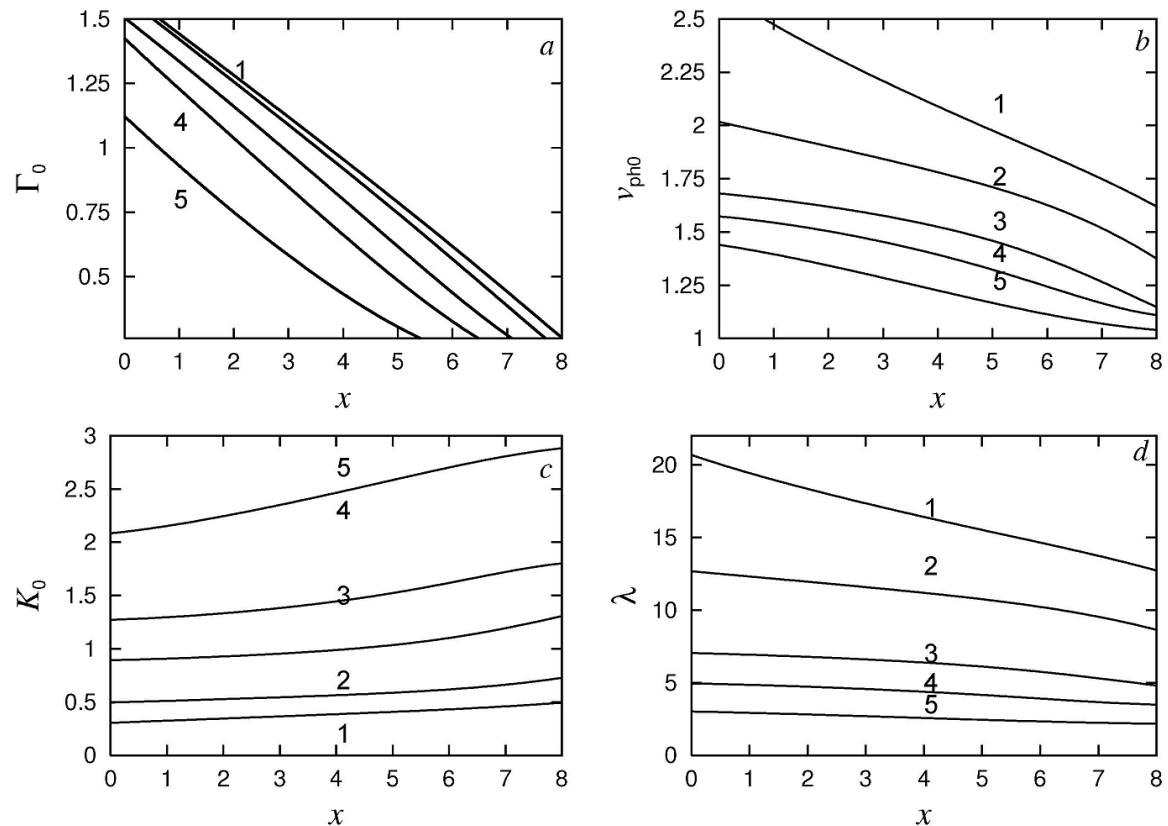

Figure 4. Dependences of the eigenvalues of the gain factor $\Gamma_{0}(a)$, wave phase velocity $v_{\text {ph0 }}(b)$, wave number $K_{0}(c)$ and wave length $\lambda(d)$ on the distance $x$ from the beam initial section for $\omega=0.8(1), \omega=1(2), \omega=1.5(3), \omega=2(4)$, and $\omega=3$ (5) 
and the formation of coherent structures, may be considered in the first approximation. They were calculated approximately for turbulent jets in [30].

\section{Conclusion}

So what has been learned? The most important result is the demonstration that the transition to turbulence and chaos in non-equilibrium inhomogeneous media arises through the simultaneous excitation of an infinite number of waves. In a sense this is unsurprising because it is already well known that a multitude of close oscillatory or wave modes can generate chaotic behavior on account of the small disturbances that are always present in any physical system [33]. But the result is of greater generality that might appear at first sight, even though the mechanism considered can only occur only in inhomogeneous systems: in reality, all systems are inhomogeneous to a greater or lesser extent, so our results are actually of global significance. They allow us to understand better the physical nature of turbulence and how it arises.

\section{Acknowledgements}

The work was supported by the Engineering and Physical Sciences Research Council and by the Royal Society (London) whom PSL gratefully acknowledges for the award of a visiting research fellowship.

\section{References}

[1] Farmer J D 1982 Physica D 4 366-393

[2] Babin A V and Vishik M I 1983 Uspekhi Matematicheskikh Nauk in Russian 38 133-187

[3] Hale J 1983 Infinite dimensional dynamical systems Lecture Notes in Mathematics vol 1007 (Berlin: Springer) pp 379-400

[4] Ilyashenko Y S 1983 Vestnik MGU Ser. 1 (mathematics, mechanics (in Russian) 24 52-59

[5] Lin C C 1955 The Theory of Hydrodynamic Stability (Cambridge: Cambridge University Press)

[6] Chandrasekhar S 1961 Hydrodynamic and Hydromagnetic Stability (Oxford: Clarendon Press)

[7] Goldshtik M A and Sctern V N 1977 Hydrodynamic Stability and Turbulence (Novosibirsk: Nauka)

[8] Drazin P D and Reid W H 1981 Hydrodynamic Stability (Cambridge: Cambridge University Press)

[9] Landau L D and Lifshitz E M 1987 Fluid Mechanics (Oxford: Butterworth and Heinemann)

[10] Monin A S and Yaglom A M 2007 Statistical Fluid Mechanics: Mechanics of Turbulence vol. 1 (Mineola NY: Dover)

[11] Qiao Z J and Li J B 2011 EPL 9450003

[12] Fisher R 1937 Ann. of Eugenics 7 355-369

[13] Kolmogorov A N, GI G I P and Piskunov N S 1937 Bulleten MGU. Matematika i Mekhanika in Russian 1 1-26

[14] Zeldovich Y B and Frank-Kamenetzki D A 1938 DAN SSSR in Russian 19 693-698

[15] Zeldovich Y B and Frank-Kamenetzki D A 1938 Zhurnal fizicheskoi khimii 12 100-105

[16] Zeldovich Y B and Raizer Y P 1966 Physics of Shock Waves and High Temperature Hydrodynamic Phenomena (Moscow: Nauka)

[17] Frank-Kamenetzky D A 1967 Diffusion and Heat-Transfer in Chemical Kinetics (Moscow: Nauka) 
[18] Zeldovich Y B, Barenblatt G I, Librovich V B and Mikhviladze G M 1980 Mathematical Burning and Explosion Theory in Russian (Moscow: Nauka)

[19] Nagumo J, Arimoto S and Yoshizawa S 1962 Proc. IRE 50 2061-2070

[20] FitzHugh R 1969 Mathematical models of excitation and propagation in nerve Biological Engeneering ed Schwan H (McGraw-Hill) pp 1-85

[21] Nicolis G 1971 Stability and dissipative structures in open systems far from equilibrium Advances in Chemical Physics vol 19 ed Prigogine I and Rice S A (New York: Wiley-Interscience) pp 209-324

[22] Ivanitsky G R, Krinsky V I and Sel'kov E E 1978 Mathematical Biophysics of the Cell (in Russian) (Moscow: Nauka)

[23] Polak L S and Mikhailov A S 1983 Self-Organization in Non-Equilibrium Physical-Chemical Systems (Moscow: Nauka)

[24] Vasilyev V A, Romanovsky Y M and Yakhno V G 1987 Auto-wave Processes (Moscow: Nauka)

[25] Landa P S 2002 J. Comms. Tech. and Electronics 47 1450-1459

[26] Rudenko O V 2011 Submerged jets, acoustic beams and flows Nonlinear Waves - 2010 ed Gaponov-Grekhov A V and Nekorkin V I (Nizhny Novgorod: Izd.IPF RAN) pp 000-000

[27] Vinogradova M B, Rudenko O V and Sukhorukov A P 1979 Theory of Waves (Moscow: Nauka)

[28] Rudenko O V and Soluyan S I 1975 Theoretical Foundations of Nonlinear Acoustics (Moscow: Nauka)

[29] Landa P S 2009 Self-oscillations in Spatially Extended Systems (Moscow: URSS)

[30] Landa P S and McClintock P V E 2004 Phys. Reports 397 1-62

[31] Mandelstam L I 1948 Complete Collected Works (Moscow: Izd-vo AN SSSR)

[32] Landa P S 1997 Discrete Dynamics in Nature and Society 1 99-110

[33] Neimark Y I and Landa P S 1992 Stochastic and Chaotic Oscillations (Dordrecht: Kluwer Academic)

[34] Ruelle D and Takens F 1971 Commun. Math. Phys. 20 167-192

[35] Nayfeh A H 1981 Introduction to Perturbation Techniques (New York: Wiley)

[36] Landa P S 1996 Nonlinear Oscillations and Waves in Dynamical Systems (Dordrecht: Kluwer)

[37] Landa P S 2010 Nonlinear Oscillations and Waves (Moscow: URSS)

[38] Waxler R 2002 J. Acoust. Soc. Amer. 112 2540-2552

[39] Neimark Y I, Kogan N Y and Savel'ev V P 1985 Dynamical Models of Control Theory (Moscow: Nauka)

[40] Korn G A and Korn T M 1968 Mathematical Handbook (New York: McGraw-Hill) 